TRANSACTIONS OF THE

AMERICAN MATHEMATICAL SOCIETY

Volume 350, Number 12, December 1998, Pages 4849-4867

S 0002-9947(98)02358-7

\title{
CHARACTERIZATIONS OF WEAKLY COMPACT OPERATORS ON $C_{o}(T)$
}

\author{
T. V. PANCHAPAGESAN
}

Dedicated to Professor V. K. Balachandran on the occasion of his seventieth birthday

\begin{abstract}
Let $T$ be a locally compact Hausdorff space and let $C_{o}(T)=\{f$ : $T \rightarrow \mathbb{C}, f$ is continuous and vanishes at infinity $\}$ be provided with the supremum norm. Let $\mathcal{B}_{c}(T)$ and $\mathcal{B}_{o}(T)$ be the $\sigma$-rings generated by the compact subsets and by the compact $G_{\delta}$ subsets of $T$, respectively. The members of $\mathcal{B}_{c}(T)$ are called $\sigma$-Borel sets of $T$ since they are precisely the $\sigma$-bounded Borel sets of $T$. The members of $\mathcal{B}_{o}(T)$ are called the Baire sets of $T . M(T)$ denotes the dual of $C_{o}(T)$. Let $X$ be a quasicomplete locally convex Hausdorff space. Suppose $u: C_{o}(T) \rightarrow X$ is a continuous linear operator. Using the Baire and $\sigma$-Borel characterizations of weakly compact sets in $M(T)$ as given in a previous paper of the author's and combining the integration technique of Bartle, Dunford and Schwartz, we obtain 35 characterizations for the operator $u$ to be weakly compact, several of which are new. The independent results on the regularity and on the regular Borel extendability of $\sigma$-additive $X$-valued Baire measures are deduced as an immediate consequence of these characterizations. Some other applications are also included.
\end{abstract}

\section{INTRODUCTION}

For a locally compact Hausdorff space $T$, let $C_{o}(T)$ be the Banach space of all continuous complex functions vanishing at infinity in $T$, endowed with the supremum norm. Let $\mathcal{B}(T)$ be the $\sigma$-algebra of Borel sets in $T$, which is the $\sigma$-algebra generated by the class of all open sets in $T$. The dual of $C_{o}(T)$ is the Banach space $M(T)$ of all bounded complex Radon measures on $T$, and by Theorem 5.3 of [Pa2] it is isometrically isomorphic to the Banach space of all Borel regular complex measures $\mu$ on $\mathcal{B}(T)$, endowed with the norm $\|\mu\|=|\mu|(T)$, where $|\mu|$ is the variation of $\mu$ in $\mathcal{B}(T)$. Hence, we identify $M(T)$ with the Banach space of all Borel regular complex measures on $\mathcal{B}(T)$. Let $X$ be a quasicomplete locally convex Hausdorff space (briefly, a quasicomplete lcHs) and let $u: C_{o}(T) \rightarrow X$ be a continuous linear map. Grothendieck gave in $[\mathrm{G}]$ some necessary and sufficient conditions for $u$ to be weakly compact.

Received by the editors November 17, 1995

1991 Mathematics Subject Classification. Primary 47B38, 46G10; Secondary 28B05.

Key words and phrases. Weakly compact operators, representing measure, vector measure, quasicomplete locally compact Hausdorff space, Borel (resp. $\sigma$-Borel, Baire) regularity, inner regularity and outer regularity.

Supported by the C.D.C.H.T. project C-586 of the Universidad de los Andes, Mérida, and by the international cooperation project between CONICIT-Venezuela and CNR-Italy.

(C)1998 American Mathematical Society 
He studied in $[\mathrm{G}]$ some topological and range properties of the adjoint $u^{*}$ and the biadjoint $u^{* *}$ of the continuous linear operator $u: C_{o}(T) \rightarrow X$, characterized weakly compact subsets of $M(T)$ and proved some deep results such as Theorems 1 and 3 and Proposition 11 of $[\mathrm{G}]$, to obtain the characterization theorem $([\mathrm{G}]$, Theorem 6 ) for weakly compact operators $u$ on $C_{o}(T)$.

Most of the results obtained in Sections 1.1, 1.2, 2.1 and 3.1 of [G] play a key role in the proof of the said characterization theorem. Moreover, the major part of the results proved in $[\mathrm{G}]$ are given only for the space $C(K)$ with $K$ compact Hausdorff, and it is remarked that the results hold also for $C_{o}(T)$ with $T$ locally compact and Hausdorff. Later, the results of Grothendieck [G] were proved in detail for the locally compact case in Sections 4.21, 4.22 and 9.1-9.4 in Edwards [E]. (In this context see Remarks 3 and 4 below.)

Recently, in $[\mathrm{Pa} 4]$ we obtained several characterizations of weakly compact sets in $M(T)$ in terms of the Baire and $\sigma$-Borel restrictions of the members of the set in question. Using these characterizations and those of Theorem 2 of $[\mathrm{G}]$ and combining the integration technique of Bartle-Dunford-Schwartz [BDS], we obtain 35 characterizations for a continuous quasicomplete lcHs-valued operator on $C_{o}(T)$ to be weakly compact. Several of them are new. The advantage of the present study is that as in [BDS] the strict Dunford-Pettis property of $C_{o}(T)$ is not needed and, in fact, it is deduced from these characterization theorems. Also the use of other deep results such as Theorem 3 and Proposition 11 of $[G]$ is dispensed with. Moreover, all the arguments are given directly for the locally compact space case, without reducing to the compact or compact metrizable case as is done in $[\mathrm{E}, \mathrm{G}]$.

Further, the isolated results on $\sigma$-additive vector valued Baire measures as in [DK, DL, K] are deduced as consequences of the present study. Also included are new proofs of Theorem 5 of [Pe] and of Theorem 5.3 (bounded Radon measure case) of $[\mathrm{Th}]$, avoiding the technique of reduction to the metrizable compact case.

\section{Preliminaries}

In this section we fix notation and terminology and also give some definitions and results from [Di], [E], [G], [Ha] and [Pa4].

Let $T$ be a locally compact Hausdorff space and let $C_{o}(T)$ be the Banach space of all complex continuous functions vanishing at infinity in $T$, endowed with the supremum norm $\|\cdot\|_{T}$. Let $\mathcal{B}(T)$ be the $\sigma$-algebra of Borel sets in $T$, which is the $\sigma$-algebra generated by the class of all open sets in $T$. Then the dual of $C_{o}(T)$ is the Banach space $M(T)$ of all bounded complex Radon measures on $T$, and by Theorem 5.3 of [Pa2] it is isometrically isomorphic to the Banach space of all Borel regular complex measures $\mu$ on $\mathcal{B}(T)$ endowed with the norm $\|\mu\|=\operatorname{var}(\mu, T)$, where the variation of $\mu$ is taken with respect to $\mathcal{B}(T)$. We denote by $\mathcal{C}(T)$ (resp. $\mathcal{C}_{o}(T)$ ) the class of all compact subsets (resp. compact $G_{\delta}$ subsets) of $T . \mathcal{B}_{c}(T)$ is the $\sigma$-ring generated by the class $\mathcal{C}(T)$, and its members are called $\sigma$-Borel sets of $T$ since they are precisely the $\sigma$-bounded Borel sets of $T$. $\mathcal{B}_{o}(T)$ is the $\sigma$-ring generated by the class $\mathcal{C}_{o}(T)$, and its members are called the Baire sets of $T$.

We recall the following lemma from $[\mathrm{Pa} 4]$ before proceeding with some definitions.

Lemma 1. For $\mu \in M(T)$, let $|\mu|(\cdot)=\operatorname{var}(\mu,(\cdot))$ in $\mathcal{B}(T)$. Then

$$
\left.|\mu|\right|_{\mathcal{B}_{o}(T)}(\cdot)=\operatorname{var}\left(\left.\mu\right|_{\mathcal{B}_{o}(T)},(\cdot)\right) \text { and }\left.|\mu|\right|_{\mathcal{B}_{c}(T)}(\cdot)=\operatorname{var}\left(\left.\mu\right|_{\mathcal{B}_{c}(T)},(\cdot)\right) \text {. }
$$


Notation 1. For $\mu \in M(T)$, let $|\mu|(\cdot)=\operatorname{var}(\mu,(\cdot))$ on $\mathcal{B}(\mathcal{T})$.

In the light of Lemma 1 , the variations used in the following definition are unambiguously defined.

Definition 1. Let $\mathcal{S}$ be a $\sigma$-ring of sets in $T$ such that $\mathcal{C}(T) \subset \mathcal{S}$ or $\mathcal{C}_{o}(T) \subset \mathcal{S}$. A complex measure $\mu$ on $\mathcal{S}$ is said to be $\mathcal{S}$-regular if, given $E \in \mathcal{S}$ and $\varepsilon>0$, there exist a compact set $K \in \mathcal{S}$ and an open set $U \in \mathcal{S}$ with $K \subset E \subset U$ such that $|\mu(B)|<\varepsilon$ for every $B \in \mathcal{S}$ with $B \subset U \backslash K$. When $\mathcal{S}=\mathcal{B}(T)$ (resp. $\mathcal{B}_{c}(T), \mathcal{B}_{o}(T)$ ), we use the terminology Borel (resp. $\sigma$-Borel, Baire) regularity in place of $\mathcal{S}$-regularity. Let $A$ be a subset of $M(T)$. We say that $A$ is uniformly Baire inner regular (resp. Baire regular) in a set $E \in \mathcal{B}_{o}(T)$ if, given $\varepsilon>0$, there exists a compact $K \in \mathcal{B}_{o}(T)$ with $K \subset E$ (resp. and an open Baire set $O$ in $T$ with $K \subset E \subset O$ ) such that $\sup _{\mu \in A}|\mu|(E \backslash K)<\varepsilon$ (resp. such that $\left.\sup _{\mu \in A}|\mu|(O \backslash K)<\varepsilon\right)$. If $A$ is uniformly Baire inner regular (resp. Baire regular) in each Baire set, then $A$ is said to be uniformly Baire inner regular (resp. Baire regular). Similarly, the uniform Borel (resp. $\sigma$-Borel) regularity and inner regularity of $A$ and those of $A$ in a Borel (resp. $\sigma$-Borel) set $E$ are defined.

By Theorem 51.D of [Ha], a compact Baire set in $T$ is a $G_{\delta}$.

It is well known that every complex Baire measure $\mu_{o}$ is Baire regular and that it has a unique extension $\mu$ to $\mathcal{B}(T)$ (resp. $\mu_{c}$ to $\mathcal{B}_{c}(T)$ ) such that $\mu$ is a Borel (resp. $\mu_{c}$ is a $\sigma$-Borel) regular complex measure. Moreover, $\mu_{\mathcal{B}_{c}(T)}=\mu_{c}$. (See, for example, Theorem 2.4 of [Pa2].)

The following proposition which is a combination of Theorems 1 and 2 of [Pa4], plays a key role in Section 5 .

Proposition 1. Let $A$ be a bounded set in $M(T)$. Then the following statements are equivalent:

(i) A is relatively weakly compact.

(ii) For each disjoint sequence $\left(U_{i}\right)$ of open Baire sets (resp. (ii)' open sets, (ii)" $\sigma$-Borel open sets) in $T, \lim _{i} \mu\left(U_{i}\right)=0$ uniformly in $\mu \in A$.

(iii) (a) A is uniformly Baire (resp. (a)' Borel, (a) " $\sigma$-Borel) inner regular in each open Baire (resp. open, $\sigma$-Borel open) set $U$ in $T$.

(b) For each $\varepsilon>0$, there exists a compact $K \in \mathcal{B}_{o}(T)$ (resp. (b)" $K \in \mathcal{C}(T)$ ) such that

$$
\sup _{\mu \in A}|\mu|(T \backslash K)<\varepsilon .
$$

(Note that $(i) \Leftrightarrow($ iii $)(a)^{\prime}$.)

(iv) $A$ is uniformly Baire (resp. (iv)' Borel, (iv)" $\sigma$-Borel) inner regular.

(v) $\left.A\right|_{\mathcal{B}_{o}(T)}$ (resp. $\left.\left.(v)^{\prime} A\right|_{\mathcal{B}(T)},\left.(v)^{\prime \prime} A\right|_{\mathcal{B}_{c}(T)}\right)$ is uniformly $\sigma$-additive.

(vi) A is uniformly Baire (resp. (vi)' Borel, (vi)" $\sigma$-Borel) regular.

The following result is also used in Section 5 .

Proposition 2. $\mathcal{B}_{o}(T)$ is also the $\sigma$-ring generated by the class of all relatively compact open Baire sets. Each open Baire set $U$ in $T$ is a countable union of compact $G_{\delta} s$ in $T$.

For a proof of the above proposition the reader can refer to $\S 14$, Chapter III of [Di]. 
A vector measure is an additive set function defined on a ring of sets with values in a lcHs. In the sequel $X$ denotes a lcHs with topology $\tau$.

The strong topology $\beta\left(X^{*}, X\right)$ of $X^{*}$ is the locally convex topology induced by the seminorms $\left\{p_{B}: B\right.$ bounded in $\left.X\right\}$, where $p_{B}\left(x^{*}\right)=\sup _{x \in B}\left|x^{*}(x)\right| . X^{* *}$ denotes the dual of $\left(X^{*}, \beta\left(X^{*}, X\right)\right)$ and is endowed with the locally convex topology $\tau_{e}$ of uniform convergence in equicontinuous subsets of $X^{*}$. Note that $\left(X^{*}, \beta\left(X^{*}, X\right)\right)$ and $\left(X^{* *}, \tau_{e}\right)$ are lcHs.

It is well known that the canonical injection $J: X \rightarrow X^{* *}$ given by $\left\langle J x, x^{*}\right\rangle=$ $\left\langle x, x^{*}\right\rangle$ for all $x \in X$ and $x^{*} \in X^{*}$ is linear. On identifying $X$ with $J X \subset X^{* *}$, one has $\tau_{e}\left|J X=\tau_{e}\right| X=\tau$.

Definition 2. A linear mapping $u: C_{o}(T) \rightarrow X$ is called a weakly compact operator on $C_{o}(T)$ if $\left\{u f:\|f\|_{T} \leq 1\right\}$ is relatively weakly compact in $X$.

Let $E$ and $F$ be lcHs and let $u: E \rightarrow F$ be a continuous linear map. Then the adjoint $u^{*}$ and the biadjoint $u^{* *}$ of $u$ are well defined linear maps with $u^{*}: F^{*} \rightarrow E^{*}$ and $u^{* *}: E^{* *} \rightarrow F^{* *}$.

Proposition 3. Let $E$ and $F$ be lcHs and let $u: E \rightarrow F$ be a continuous linear map. Then $u^{*}:\left(F^{*}, \sigma\left(F^{*}, F\right)\right) \rightarrow\left(E^{*}, \sigma\left(E^{*}, E\right)\right)$ is continuous and $u^{* *}$ : $\left(E^{* *}, \tau_{e}\right) \rightarrow\left(F^{* *}, \tau_{e}\right)$ is continuous.

See the corollary to Proposition $1, \S 12$, Chapter 3 of [Ho] and Proposition 8.7.2 of $[\mathrm{E}]$.

The following result (Corollary 9.3.2 of [E], which is essentially due to Lemma 1 of $[\mathrm{G}])$ is indispensable in the study of weakly compact operators on $C_{o}(T)$ in Section 5 .

Proposition 4. Let $E$ and $F$ be lcHs with $F$ quasicomplete. If $u: E \rightarrow F$ is linear and continuous, then $u$ maps bounded subsets of $E$ into relatively weakly compact subsets of $F$ if and only if $u^{*}(A)$ is relatively $\sigma\left(E^{*}, E^{* *}\right)$-compact for each equicontinuous subset $A$ of $F^{*}$.

\section{Integration of Bounded $\mathcal{S}$-MeAsurable SCALAR FUnCTIONS}

In this section we define the integral of a bounded $\mathcal{S}$-measurable scalar function with respect to an $X$-valued vector measure $m$ defined on a $\sigma$-ring $\mathcal{S}$, where $m$ is either $\sigma$-additive or bounded and $X$ is a quasicomplete lcHs. To this end, we introduce the following additional notation and terminology.

For each $\tau$-continuous seminorm $p$ on $X$, let $p(x)=\|x\|_{p}, x \in X$, and let $X_{p}=\left(X,\|\cdot\|_{p}\right)$ be the associated seminormed space. The completion of the quotient normed space $X_{p} / p^{-1}(0)$ is denoted by $\tilde{X}_{p}$. Let $\Pi_{p}: X_{p} \rightarrow X_{p} / p^{-1}(0) \subset \tilde{X}_{p}$ be the canonical quotient map.

Let $\mathcal{S}$ be a $\sigma$-ring of subsets of a nonempty set $\Omega$. Given a vector measure $m: \mathcal{S} \rightarrow X$, for each $\tau$-continuous seminorm $p$ on $X$ let $m_{p}: \mathcal{S} \rightarrow \tilde{X}_{p}$ be given by $m_{p}(E)=\Pi_{p} \circ m(E)$ for $E \in \mathcal{S}$. Then $m_{p}$ is a Banach space valued vector measure on $\mathcal{S}$. We define the $p$-semivariation $\|m\|_{p}$ of $m$ by

$$
\|m\|_{p}(E)=\left\|m_{p}\right\|(E) \text { for } E \in \mathcal{S}
$$

and

$$
\|m\|_{p}(\Omega)=\left\|m_{p}\right\|(\Omega)=\sup _{E \in \mathcal{S}}\left\|m_{p}\right\|(E)
$$


where $\left\|m_{p}\right\|$ is the semivariation of the vector measure $m_{p}: \mathcal{S} \rightarrow \tilde{X}_{p}$. When $m$ is $\sigma$-additive, then $m_{p}$ is a Banach space valued $\sigma$-additive vector measure, and hence, by a well known result on vector measures, $\|m\|_{p}(\Omega)=\left\|m_{p}\right\|(\Omega)<\infty$.

An $X$-valued vector measure $m$ on a $\sigma$-ring $\mathcal{S}$ of subsets of $\Omega$ is said to be bounded if for each continuous seminorm $p$ on $X,\|m\|_{p}(\Omega)<\infty$.

For an $\mathcal{S}$-simple scalar function $s=\sum_{i=1}^{r} \lambda_{i} \chi_{E_{i}}, \lambda_{i} \neq 0, E_{i} \in \mathcal{S}, E_{i} \cap E_{j}=\emptyset$ for $i \neq j, i, j=1,2, \ldots, r$, and for an lcHs-valued bounded vector measure $m$ we define

$$
\int_{E} s d m=\sum_{i=1}^{r} \lambda_{i} m\left(E \cap E_{i}\right)
$$

for $E \in \mathcal{S}$. It is easy to verify that $\int_{E} s d m$ is well defined, and for each $E$ fixed in $\mathcal{S}$ it is linear on the normed space $S(\mathcal{S})$ of all $\mathcal{S}$-simple scalar functions endowed with the supremum norm $\|\cdot\|_{\Omega}$.

Proposition 5. Let $\left(s_{n}\right)$ and $\left(s_{n}^{\prime}\right)$ be sequences of $\mathcal{S}$-simple scalar functions, converging uniformly to a function $f$ in $\Omega$. Suppose $m: \mathcal{S} \rightarrow X$ is $\sigma$-additive or is a bounded vector measure, where $X$ is a quasicomplete lcHs. Then:

(i) $\left(\int_{E} s_{n} d m\right)$ is uniformly Cauchy in $X$ for $E \in \mathcal{S}$.

(ii) $\lim _{n} \int_{E} s_{n} d m=\lim _{n} \int_{E} s_{n}^{\prime} d m \in X$ for each $E \in \mathcal{S}$.

Proof. Let $p$ be a $\tau$-continuous seminorm on $X$ and let $\varepsilon>0$. Since $\left\|m_{p}\right\|(\Omega)<\infty$, we can choose $n_{o}$ such that $\left\|s_{n}-s_{\ell}\right\|_{\Omega}<\frac{\varepsilon}{\left\|m_{p}\right\|(\Omega)}$ for $n, \ell \geq n_{o}$. Then

$$
\left\|\int_{E} s_{n} d m-\int_{E} s_{\ell} d m\right\|_{p}=\left\|\int_{E}\left(s_{n}-s_{\ell}\right) d m_{p}\right\|_{p} \leq\left\|s_{n}-s_{\ell}\right\|_{\Omega}\left\|m_{p}\right\|(\Omega)<\varepsilon
$$

for $n, \ell \geq n_{o}$ and for all $E \in \mathcal{S}$. Hence (i) holds.

As $X$ is sequentially complete, by (i) there exist vectors $x_{E}, x_{E}^{\prime}$ in $X$ such that $\lim _{n} \int_{E} s_{n} d m=x_{E}$ and $\lim _{n} \int_{E} s_{n}^{\prime} d m=x_{E}^{\prime}$ for $E \in \mathcal{S}$. Then

$$
\begin{aligned}
\left\|x_{E}-x_{E}^{\prime}\right\|_{p} \leq & \left\|\int_{E} s_{n} d m-x_{E}\right\|_{p}+\left\|\int_{E} s_{n} d m-\int_{E} s_{n}^{\prime} d m\right\|_{p} \\
& +\left\|\int_{E} s_{n}^{\prime} d m-x_{E}^{\prime}\right\|_{p} \rightarrow 0
\end{aligned}
$$

as $n \rightarrow \infty$, since

$$
\left\|\int_{E} s_{n} d m-\int_{E} s_{n}^{\prime} d m\right\|_{p} \leq\left\|s_{n}-s_{n}^{\prime}\right\|_{\Omega}\left\|m_{p}\right\|(\Omega) \rightarrow 0
$$

as $n \rightarrow \infty$. As the $\tau$-continuous seminorm $p$ is arbitrary, it follows that $x_{E}=x_{E}^{\prime}$ for each $E \in \mathcal{S}$. Hence (ii) holds.

Definition 3. Let $f$ be a bounded $\mathcal{S}$-measurable scalar function so that there exists a sequence $\left(s_{n}\right)$ of $\mathcal{S}$-simple scalar functions such that $s_{n} \rightarrow f$ uniformly in $\Omega$. If $m: \mathcal{S} \rightarrow X$ is $\sigma$-additive or is additive and bounded, and $X$ is a quasicomplete lcHs, then we say that $f$ is $m$-integrable and define

$$
\int_{E} f d m=\lim _{n} \int_{E} s_{n} d m
$$

for each $E \in \mathcal{S}$. 
In the light of Proposition 5, for a bounded $\mathcal{S}$-measurable scalar function $f$, $\int_{E} f d m$ is well defined. Since $N(f)=\{w \in \Omega: f(w) \neq 0\} \in \mathcal{S}$, we then define

$$
\int_{\Omega} f d m=\int_{N(f)} f d m
$$

The following proposition is immediate from Definition 3.

Proposition 6. Let $X$ be a quasicomplete lcHs. If $f$ and $g$ are bounded $\mathcal{S}$-measurable scalar functions, $\alpha, \beta$ are scalars, $m: \mathcal{S} \rightarrow X$ is $\sigma$-additive or bounded and additive, and $E \in \mathcal{S}$, then the following hold:

(i) $\int_{E}(\alpha f+\beta g) d m=\alpha \int_{E} f d m+\beta \int_{E} g d m$.

(ii) $\left\|\int_{E} f d m\right\|_{p} \leq\|f\|_{E}\left\|m_{p}\right\|(E) \leq\|f\|_{\Omega}\left\|m_{p}\right\|(\Omega)=\|f\|_{\Omega}\|m\|_{p}(\Omega)$ for each $\tau$-continuous seminorm $p$ on $X$.

(iii) For each $x^{*} \in X^{*}, x^{*}\left(\int_{E} f d m\right)=\int_{E} f d\left(x^{*} m\right)$.

Proposition 7 (Lebesgue bounded convergence theorem). Let $X$ be a quasicomplete lcHs and let $m: \mathcal{S} \rightarrow X$ be $\sigma$-additive. If $\left(f_{n}\right)$ is a bounded sequence of $\mathcal{S}$-measurable scalar functions with $\lim _{n} f_{n}(w)=f(w)$ for each $w \in \Omega$, then $f$ is $m$-integrable and

$$
\int_{E} f d m=\lim _{n} \int_{E} f_{n} d m
$$

for each $E \in \mathcal{S}$.

Proof. For each $E \in \mathcal{S}, \mathcal{S}_{E}=\mathcal{S} \cap E$ is a $\sigma$ - algebra in $E$. Then for each $\tau$-continuous seminorm $p$ on $X$, by Theorem II.4.1 of [DU], we have

$$
\left\|\int_{E} f_{n} d m-\int_{E} f d m\right\|_{p}=\left\|\int_{E} f_{n} d m_{p}-\int_{E} f d m_{p}\right\|_{p} \rightarrow 0
$$

as $n \rightarrow \infty$. Hence the proposition holds.

Remark 1. Using the Egoroff-Lusin theorem for continuous submeasures, one can also show that the limit in the above proposition is uniform with respect to $E \in \mathcal{S}$. (See Theorem 1 of [Do].)

\section{Representation of $X$-valued continuous LINEAR TRANSFORMATIONS ON $C_{o}(T)$}

The following result is analogous to Theorem VI.2.1 of [DU] for a lcHs-valued continuous linear map on $C_{o}(T)$, and plays a fundamental role in Sections 5 and 6 .

Theorem 1. Let $X$ be an lcHs. Let $u: C_{o}(T) \rightarrow X$ be a continuous linear transformation. Then there exists an $X^{* *}$-valued vector measure $m$ on $\mathcal{B}(T)$ with the following properties:

(i) $x^{*} m \in M(T)$ for each $x^{*} \in X^{*}$, and consequently $m: \mathcal{B}(T) \rightarrow X^{* *}$ is $\sigma$ additive in the $\sigma\left(X^{* *}, X^{*}\right)$-topology.

(ii) The mapping $x^{*} \rightarrow x^{*} m$ of $X^{*}$ into $M(T)$ is weak*-weak* continuous.

(iii) $x^{*} u f=\int_{T} f d x^{*} m$ for each $f \in C_{o}(T)$ and $x^{*} \in X^{*}$.

(iv) $\{m(E): E \in \mathcal{B}(T)\}$ is $\tau_{e}$-bounded in $X^{* *}$.

Conversely, let $m: \mathcal{B}(T) \rightarrow X^{* *}$ be a vector measure satisfying (i) and (ii). Then there exists a unique continuous linear transformation $u: C_{o}(T) \rightarrow X$ such that (iii) holds. Moreover, $m(E)=u^{* *}\left(\chi_{E}\right)$ for $E \in \mathcal{B}(T)$ and $m$ satisfies (iv). 
Finally, the vector measure $m$ satisfying (i)-(iii) is uniquely determined by the continuous linear transformation $u$ and has $\tau_{e}$-bounded range in $X^{* *}$.

Proof. Note that $u^{*}: X^{*} \rightarrow M(T)$ and $u^{* *}: C_{o}^{* *}(T) \rightarrow X^{* *}$ are linear. Since each bounded Borel measurable scalar function $f$ on $T$ can be identified with a unique element $\imath(f) \in C_{o}^{* *}(T)$ such that $\|f\|_{T}=\|\imath(f)\|$, we shall identify $\left\{\chi_{E}: E \in \mathcal{B}(T)\right\}$ as a subset of $C_{o}^{* *}(T)$. Let us define $m: \mathcal{B}(T) \rightarrow X^{* *}$ by putting $m(E)=u^{* *}\left(\chi_{E}\right)$ for $E \in \mathcal{B}(T)$. Then $m$ is clearly additive. Moreover, $u^{*} x^{*} \in M(T)$ for each $x^{*} \in X^{*}$ and hence there exists a unique $\mu_{x^{*}} \in M(T)$ such that $u^{*} x^{*}=\mu_{x^{*}}$. Consequently,

$$
\mu_{x^{*}}(E)=\left(u^{*} x^{*}\right)(E)=\left\langle u^{*} x^{*}, \chi_{E}\right\rangle=\left\langle x^{*}, u^{* *}\left(\chi_{E}\right)\right\rangle=\left\langle x^{*}, m(E)\right\rangle=\left(x^{*} m\right)(E)
$$

for each $E \in \mathcal{B}(T)$. Hence $\mu_{x^{*}}=u^{*} x^{*}=x^{*} m$ for $x^{*} \in X^{*}$. Thus (i) holds.

By the first part of Proposition 3,

$$
u^{*}:\left(X^{*}, \sigma\left(X^{*}, X\right)\right) \rightarrow\left(M(T), \sigma\left(M(T), C_{o}(T)\right)\right)
$$

is continuous, whence (ii) follows.

Since

$$
x^{*}(u f)=\left\langle u f, x^{*}\right\rangle=\left\langle f, u^{*} x^{*}\right\rangle=\left\langle f, x^{*} m\right\rangle=\int_{T} f d\left(x^{*} m\right)
$$

for each $f \in C_{o}(T)$ and $x^{*} \in X^{*}$, (iii) holds.

By the second part of Proposition 3 and by Theorem 1.32 of Rudin $[\mathrm{R}],\{m(E)$ : $E \in \mathcal{B}(T)\}=\left\{u^{* *}\left(\chi_{E}\right): E \in \mathcal{B}(T)\right\}$ is $\tau_{e}$-bounded in $X^{* *}$, and hence (iv) holds.

Conversely, let $m: \mathcal{B}(T) \rightarrow X^{* *}$ be a vector measure satisfying (i) and (ii). Let $x^{*} \in X^{*}$. Then by (i) there exists $\mu_{x^{*}} \in M(T)$ such that $x^{*} m=\mu_{x^{*}}$, and by (ii) the mapping $V: X^{*} \rightarrow M(T)$ given by $V x^{*}=\mu_{x^{*}}$ is linear and weak*weak* continuous. For $f \in C_{o}(T)$, let $V_{f}: X^{*} \rightarrow \mathbb{C}$ be given by $V_{f}\left(x^{*}\right)=$ $\left\langle f, V x^{*}\right\rangle=\int_{T} f d \mu_{x^{*}}$. Then by the weak*-weak ${ }^{*}$ continuity of $V$ it follows that $V_{f}$ is a $\sigma\left(X^{*}, X\right)$-continuous linear functional on $X^{*}$ for each $f \in C_{o}(T)$. Consequently, there exists a unique vector $x_{f} \in X$ such that $V_{f} x^{*}=x^{*}\left(x_{f}\right)$ for each $x^{*} \in X^{*}$. Let $u f=x_{f}, f \in C_{o}(T)$. Clearly, $u: C_{o}(T) \rightarrow X$ is linear. Moreover,

$$
\begin{aligned}
\sup _{\|f\|_{T} \leq 1}\left|x^{*} u f\right| & =\sup _{\|f\|_{T} \leq 1}\left|x^{*} x_{f}\right|=\sup _{\|f\|_{T} \leq 1}\left|V_{f} x^{*}\right| \\
& =\sup _{\|f\|_{T} \leq 1}\left|\int_{T} f d\left(x^{*} m\right)\right| \leq\left|x^{*} m\right|(T)<\infty
\end{aligned}
$$

for each $x^{*} \in X^{*}$. Consequently, $\left\{u f:\|f\|_{T} \leq 1\right\}$ is weakly bounded in $X$ and hence $\tau$-bounded by Theorem 3.18 of Rudin [R]. Then by Theorem 1.32 of Rudin $[\mathrm{R}], u$ is continuous. Moreover,

$$
x^{*} u f=x^{*} x_{f}=V_{f} x^{*}=\int_{T} f d\left(x^{*} m\right)
$$

for each $f \in C_{o}(T)$ and $x^{*} \in X^{*}$. Thus the continuous linear transformation $u$ satisfies (iii).

If there exists another continuous linear transformation $v: C_{o}(T) \rightarrow X$ such that $x^{*} v f=\int_{T} f d\left(x^{*} m\right)$ for each $x^{*} \in X^{*}$ and $f \in C_{o}(T)$, then by (4.1) and by the Hahn-Banach theorem we have $v=u$.

Let $m_{1}$ be the vector measure defined on $\mathcal{B}(T)$ by $m_{1}(E)=u^{* *}\left(\chi_{E}\right)$. Then as shown in the proof of the first part we have $u^{*} x^{*}=x^{*} m_{1}$ for each $x^{*} \in X^{*}$, 
$x^{*} u f=\int_{T} f d\left(x^{*} m_{1}\right)$ for $f \in C_{o}(T)$ and $x^{*} \in X^{*}$, and $m_{1}$ has $\tau_{e}$-bounded range in $X^{* *}$. Then by (4.1) we have

$$
x^{*} u f=\int_{T} f d\left(x^{*} m_{1}\right)=\int_{T} f d\left(x^{*} m\right)
$$

for $f \in C_{o}(T)$. Since $x^{*} m, x^{*} m_{1}$ are regular complex Borel measures on $T$, by the uniqueness part of the Riesz representation theorem it follows that $x^{*} m=x^{*} m_{1}$ on $\mathcal{B}(T)$ for each $x^{*} \in X^{*}$, and hence $m_{1}=m$. Consequently, $m$ has $\tau_{e}$-bounded range.

Definition 4. Given a continuous linear transformation $u: C_{o}(T) \rightarrow X$, the unique $X^{* *}$-valued vector measure $m$ satisfying (i)-(iii) of Theorem 1 is called the representing measure of $u$.

\section{Characterizations of $X$-valued WEAKLY COMPACT OPERATORS ON $C_{o}(T)$}

Making use of the results of the earlier sections, we obtain 35 characterizations of a quasicomplete lcHs-valued weakly compact operator on $C_{o}(T)$. Among them are Gothendieck's characterizations from Remark 2 of [G], as well as several new ones. For convenience of exposition we give these characterizations through eight theorems, instead of all in a single theorem. The equivalent statements given in these theorems are numbered successively, and they will be referred to as (viii), (xii), etc. without mentioning the theorem in which they appear.

Motivated by Definition 3.2 of [Pa1], we give the following notions of regularity for a vector measure.

Definition 5. Let $\mathcal{R}$ be a ring of sets in $T$ with $\mathcal{R} \supset \mathcal{C}(T)$ or $\mathcal{R} \supset \mathcal{C}_{o}(T)$. Let $m: \mathcal{R} \rightarrow X$ be a vector measure. Then $m$ is said to be $\mathcal{R}$-regular or simply regular (resp. $\mathcal{R}$-outer regular or simply outer regular, $\mathcal{R}$-inner regular or simply inner regular) in $E \in \mathcal{R}$ if, given $\varepsilon>0$ and a $\tau$-continuous seminorm $p$ on $X$, there exist a compact set $K \in \mathcal{R}$ and an open set $U \in \mathcal{R}$ with $K \subset E \subset U$ (resp. there exists an open set $U \in \mathcal{R}$ with $E \subset U$, there exists a compact set $K \in \mathcal{R}$ with $K \subset E$ ) such that for each $B \in \mathcal{R}$ with $B \subset U \backslash K$ (resp. with $B \subset U \backslash E$, with $B \subset E \backslash K)$ we have $p(m(B))<\varepsilon$. Even though $T$ does not belong to $\mathcal{R}$, one can define $\mathcal{R}$-inner regularity of $m$ in $T$ on similar lines. The vector measure $m$ is said to be $\mathcal{R}$-regular or simply regular (resp. $\mathcal{R}$-outer regular or simply outer regular, $\mathcal{R}$-inner regular or simply inner regular) if it is so in each $E \in \mathcal{R}$. When $\mathcal{R}=\mathcal{B}(T)$ (resp. $\mathcal{B}_{c}(T), \mathcal{B}_{o}(T)$ ), we use the terminolgy Borel (resp. $\sigma$-Borel, Baire) regularity or outer regularity or inner regularity.

Remark 2. In Definition 5 one can restrict to any family of $\tau$-continuous seminorms on $X$ which induces the topology $\tau$ in $X$.

Notation 2. For a set $E$ in $X^{*}, p_{E}\left(x^{* *}\right)=\sup _{x^{*} \in E}\left|x^{* *}\left(x^{*}\right)\right|, x^{* *} \in X^{* *}$.

Lemma 2. Let $X$ be a quasicomplete lcHs and let $u: C_{o}(T) \rightarrow X$ be a continuous linear map. Then $u^{*} E$ is bounded in $M(T)$ for each equicontinuous set $E$ in $X^{*}$.

Proof. Let $m$ be the representing measure of $u$. Then from the proof of Theorem 1(i) we have $u^{*} E=\left\{x^{*} \circ m: x^{*} \in E\right\}$. As $m$ has $\tau_{e}$-bounded range by Theorem 
1 (iv), it follows that

$$
\sup _{x^{*} \in E}\left|x^{*} \circ m\right|(T) \leq 4 \sup _{x^{*} \in E, A \in \mathcal{B}(T)}\left|\left(x^{*} \circ m\right)(A)\right|=4 \sup _{A \in \mathcal{B}(T)} p_{E}(m(A))<\infty .
$$

Thus $u^{*} E$ is bounded in $M(T)$.

Theorem 2. Let $u: C_{o}(T) \rightarrow X$ be a continuous linear transformation, where $X$ is a quasicomplete lcHs, and let $m: \mathcal{B}(T) \rightarrow X^{* *}$ be the representing measure of $u$. Then the following statements are equivalent:

(i) $u$ is weakly compact.

(ii) The range of $m$ is contained in $X$.

(iii) $m$ is $\sigma$-additive in the topology $\tau_{e}$ of $X^{* *}$.

Proof. (i) $\Rightarrow$ (ii). By the equivalence of (1) and (2) of Lemma 1 of Grothendieck $[\mathrm{G}], u$ is weakly compact if and only if $u^{* *}\left(C_{o}^{* *}(T)\right) \subset X$. Since $m(E)=u^{* *}\left(\chi_{E}\right)$ for $E \in \mathcal{B}(T)$, (i) $\Rightarrow$ (ii). (Note that the quasicompleteness of $X$ is not needed for this implication.)

(ii) $\Rightarrow$ (iii). By Theorem 1(i) and the hypothesis (ii), the vector measure $m$ is $\sigma$ additive in $\sigma\left(X, X^{*}\right)$-topology. Since $\left.\tau_{e}\right|_{X}=\tau$, now (iii) holds by the Orlicz-Pettis theorem (see, for example, $[\mathrm{M}]$ ).

(iii) $\Rightarrow$ (i). Let $\left(U_{n}\right)$ be a disjoint sequence of open sets in $T$ and let $E$ be an equicontinuous subset of $X^{*}$. If $U=\bigcup_{n=1}^{\infty} U_{n}$, then (iii) implies that $\left\|m\left(U_{n}\right)\right\|_{p_{E}} \rightarrow$ 0 as $n \rightarrow \infty$. In other words, $\lim _{n} x^{*} \circ m\left(U_{n}\right)=0$ uniformly in $x^{*} \in E$. Since $u^{*} E$ is bounded in $M(T)$ by Lemma 2 , and since $u^{*} x^{*}=x^{*} \circ m$, by the equivalence of (i) and (ii) ${ }^{\prime}$ of Proposition 1 it follows that $u^{*} E$ is relatively weakly compact in $M(T)$. Then by Proposition $4, u$ is weakly compact and hence (i) holds.

Theorem 3. Let $X, u$ and $m$ be as in Theorem 2. Then $u$ is weakly compact if and only if any one of the following conditions holds:

(iv) $m(U) \in X$ for all open sets $U$ in $T$.

(v) $m(F) \in X$ for all closed sets $F$ in $T$.

(vi) $m(U) \in X$ for all open sets $U$ in $T$ which are $\sigma$-Borel.

(vii) $m(U) \in X$ for all open Baire sets $U$ in $T$.

(viii) $m(U) \in X$ for all open sets $U$ in $T$ which are $\sigma$-compact in $T$.

(ix) $m(F) \in X$ for all closed sets $F$ in $T$ which are $G_{\delta}$.

(x) $m(U) \in X$ for all open sets $U$ in $T$ which are a countable union of closed sets in $T$.

(xi) For each increasing sequence $\left(f_{n}\right)_{1}^{\infty} \subset C_{0}(T)$, with $0 \leq f_{n} \leq 1,\left(u f_{n}\right)$ converges weakly in $X$.

Proof. Obviously (iv) $\Rightarrow$ (v) $\Rightarrow$ (ix); (iv) $\Rightarrow$ (vi) $\Rightarrow$ (vii); (iv) $\Rightarrow$ (viii); (iv) $\Rightarrow$ (x) and $(\mathrm{x}) \Rightarrow$ (viii). Moreover, (viii) $\Rightarrow$ (vii) by Proposition 2 and (i) $\Rightarrow$ (iv) by Theorem 2 .

(vii) $\Rightarrow$ (i). Let $\left(U_{n}\right)$ be a disjoint sequence of open Baire sets in $T$ and let $U=\bigcup_{n=1}^{\infty} U_{n}$. Then by Theorem 1(i), the hypothesis (vii) and the Orlicz-Pettis theorem, $m(U)=\sum_{n=1}^{\infty} m\left(U_{n}\right)$ in the topology $\tau$ of $X$. Let $E$ be an equicontinuous set in $X^{*}$. Since $\left.\tau_{e}\right|_{X}=\tau$, it follows that $\left\|m\left(U_{n}\right)\right\|_{p_{E}} \rightarrow 0$. Then the argument in the proof of (iii) $\Rightarrow$ (i) of Theorem 2 holds here verbatim except that we have to invoke the equivalence of (i) and (ii) of Proposition 1 instead of that of (i) and (ii)' of Proposition 1. Hence $u$ is weakly compact.

(ix) $\Rightarrow$ (vii). Let $U$ be an open Baire set in $T$. Then by Proposition $2, U$ is of the form $U=\bigcup_{n=1}^{\infty} K_{n}$ with $K_{n} \in \mathcal{C}_{o}(T)$ for each $n$. Then $T \backslash U=\bigcap_{n=1}^{\infty}\left(T \backslash K_{n}\right)$ 
is a $G_{\delta}$. Thus (ix) implies that $m(T)$ and $m(T \backslash U)$ belong to $X$. Then $m(U)=$ $m(T)-m(T \backslash U) \in X$, and hence (vii) holds.

(vii) $\Rightarrow$ (viii) since (iv) $\Leftrightarrow$ (vii) and (iv) $\Rightarrow$ (viii).

(viii) $\Rightarrow$ (x) since (viii) $\Rightarrow$ (vii) $\Leftrightarrow$ (iv), and (iv) $\Rightarrow$ (x).

(i) $\Rightarrow(\mathrm{xi})$. Let $\left(f_{n}\right)_{1}^{\infty}$ be an increasing sequence in $C_{o}(T)$, with $0 \leq f_{n} \leq 1$. Clearly, $\lim _{n} f_{n}(t)=f(t)$ exists in $[0,1]$ for each $t \in T$, and moreover, $f$ is Borel measurable. Then by Proposition 7, Theorem 2 and the fact that $\left.\tau_{e}\right|_{X}=\tau$ we have

$$
\lim _{n} \int_{T} f_{n} d m=\int_{T} f d m \in X
$$

in the topology $\tau$ of $X$. Consequently, by Theorem 1(iii) and Proposition 6(iii)

$$
\lim _{n} x^{*} u f_{n}=\lim _{n} \int_{T} f_{n} d\left(x^{*} m\right)=x^{*}\left(\int_{T} f d m\right)
$$

for each $x^{*} \in X^{*}$. Hence (xi) holds.

(xi) $\Rightarrow$ (vii). Let $U$ be an open Baire set in $T$. Then by Proposition $2, U$ is the union of an increasing sequence $\left(K_{n}\right)$ of compact $G_{\delta}$ subsets of $T$. Then by Urysohn's lemma there exists an increasing sequence $\left(g_{n}\right)_{1}^{\infty}$ of nonnegative continuous functions with compact support such that $g_{n} \nearrow \chi_{U}$. Therefore, by hypothesis there exists a vector $x_{o} \in X$ such that $x^{*} u g_{n} \rightarrow x^{*}\left(x_{o}\right)$ for each $x^{*} \in X^{*}$. On the other hand, by Theorem 1(iii) and by the Lebesgue dominated convergence theorem we have

$$
\lim _{n} x^{*} u g_{n}=\lim _{n} \int_{T} g_{n} d\left(x^{*} m\right)=\int_{T} \chi_{U} d\left(x^{*} m\right)=\left(x^{*} m\right)(U)
$$

for each $x^{*} \in X^{*}$. Thus $x^{*}\left(m(U)-x_{o}\right)=0$ for each $x^{*} \in X^{*}$. Since $x_{o} \in X$ and $m(U) \in X^{* *}$, it follows that $m(U)=x_{o} \in X$. Hence (vii) holds.

Remark 3. An alternative proof of $(\mathrm{i}) \Rightarrow$ (xi) without using Proposition 7 is given in Grothendieck [G]. Since $\left(f_{n}\right)$ is weakly Cauchy, $u$ is weakly compact and $C_{o}(T)$ has the strict Dunford-Pettis property, it follows that $\left(u f_{n}\right)$ is Cauchy in the quasicomplete lcHs $X$ and hence $\left(u f_{n}\right)$ converges in the topology $\tau$ of $X$. Thus, in particular, it is weakly convergent. Our proof does not use the strict DunfordPettis property of $C_{o}(T)$, which will later be deduced in Section 6 (see Theorem 11) as a consequence of Theorems 1 and 2 and Proposition 7. In this connection we would also like to point out that the argument in the proof of (1) implies (3) of Theorem 9.4.10 of Edwards [E] is incorrect and has to be replaced as above, invoking the strict Dunford-Pettis property of $C_{o}(T)$.

Remark 4. When $T$ is compact and Hausdorff, Grothendieck proved in [G] the equivalence of the statements (i), (v), (ix) and (xi) (see Theorem 6 of $[\mathrm{G}]$ ). Then he commented in Remark 2 on p.161 of [G] that the theorem holds also for locally compact Hausdorff spaces, but did not provide details. Later, Edwards provided details for the general case in the proof of Theorem 9.4.10 of [E], but unfortunately his proof of $(3) \Rightarrow(2$ bis $)$ is incorrect. However, Theorems 2 and 3 above establish its validity in the general case. Moreover, even for the compact case, Grothendieck's proof needs his deep results such as Theorem 3 and Proposition 11 of $[\mathrm{G}]$. On the other hand, our proof given here is not only more elementary but also is applicable to the general locally compact case. 
Theorem 4. Let $X, u$ and $m$ be as in Theorem 2. Let $m_{c}=\left.m\right|_{\mathcal{B}_{c}(T)}$ and $m_{o}=$ $\left.m\right|_{\mathcal{B}_{o}(T)}$. Then $u$ is weakly compact if and only if any one of the following equivalent statements holds:

(xii) $m_{c}$ is $\sigma$-additive in the topology $\tau_{e}$ of $X^{* *}$.

(xiii) $m_{o}$ is $\sigma$-additive in the topology $\tau_{e}$ of $X^{* *}$.

(xiv) $m_{c}$ has range in $X$.

(xv) $m_{o}$ has range in $X$.

Proof. In the light of Theorem 2, it suffices to show that (xiii) $\Rightarrow$ (i) and (xv) $\Rightarrow$ (xiii).

(xiii) $\Rightarrow$ (i). Let $\left(U_{n}\right)$ be a disjoint sequence of open Baire sets in $T$ with $U=$ $\bigcup_{1}^{\infty} U_{n}$. Then, by hypothesis (xiii), $m_{o}(U)=\sum_{1}^{\infty} m_{o}\left(U_{n}\right)$ in the topology $\tau_{e}$ of $X^{* *}$, and hence $\lim _{n} m_{o}\left(U_{n}\right)=0$ in $\tau_{e}$ of $X^{* *}$. Thus, for each equicontinuous set $E$ in $X^{*},\left\|m_{o}\left(U_{n}\right)\right\|_{p_{E}} \rightarrow 0$ as $n \rightarrow \infty$. In other words, $\lim _{n}\left(x^{*} \circ m_{o}\right)\left(U_{n}\right)=0$ uniformly in $x^{*} \in E$. Since $u^{*} x^{*}=x^{*} \circ m$ and since $u^{*} E$ is bounded in $M(T)$ by Lemma 2, by the equivalence of (i) and (ii) of Proposition 1 it follows that $u^{*} E$ is relatively weakly compact in $M(T)$. Now by Proposition 4 we conclude that $u$ is weakly compact.

(xv) $\Rightarrow$ (xiii). Since $m_{o}$ is $\sigma$-additive in the $\sigma\left(X^{* *}, X^{*}\right)$-topology by Theorem (i) and since $m_{o}$ has range in $X$, by the Orlicz-Pettis theorem $m_{o}$ is $\sigma$-additive in the topology $\tau$ of $X$. Since $\left.\tau_{e}\right|_{X}=\tau$, the statement (xiii) holds.

Let us recall that a vector measure $\nu$ defined on a ring of sets $\mathcal{R}$ with values in a lcHs $X$ is said to be strongly additive if for each disjoint sequence $\left(E_{n}\right) \subset \mathcal{R}$ we have $\lim _{n} \nu\left(E_{n}\right)=0$. Since in the proof of (xiii) $\Rightarrow$ (i) we have used only the fact that $\lim _{n} m_{o}\left(U_{n}\right)=0$ for a disjoint sequence of open Baire sets $\left(U_{n}\right)$ in $T$, and since $\sigma$-additivity in a $\sigma$-ring implies strong additivity, the following theorem is immediate.

Theorem 5. Let $X, u, m, m_{c}$ and $m_{o}$ be as in Theorem 4. Then $u$ is weakly compact if and only if any one of the following conditions holds:

(xvi) $m$ is strongly additive in the topology $\tau_{e}$ of $X^{* *}$.

(xvii) $m_{c}$ is strongly additive in the topology $\tau_{e}$ of $X^{* *}$.

(xviii) $m_{o}$ is strongly additive in the topology $\tau_{e}$ of $X^{* *}$.

Theorem 6. Let $X, u$ and $m$ be as in Theorem 2. Then $u$ is weakly compact if and only if any one of the following conditions holds, where the regularities referred to are with respect to the topology $\tau_{e}$ of $X^{* *}$.

(xix) $m$ is Borel regular.

$(\mathrm{xx}) m$ is Borel inner regular.

(xxi) $m$ is Borel inner regular in each open set in $T$.

(xxii) $m$ is Borel outer regular in each compact set in $T$ and Borel inner regular in the set $T$.

Proof. (i) $\Rightarrow$ (xix). Let $A \in \mathcal{B}(T), \varepsilon>0$, and $E$ an equicontinuous set in $X^{*}$. It suffices to show that there exist a compact $K$ and an open set $U$ in $T$ with $K \subset A \subset U$ such that $p_{E}(m(B))<\varepsilon$ for all $B \in \mathcal{B}(T)$ with $B \subset U \backslash K$. Since $u$ is weakly compact, by Proposition $4 u^{*} E$ is relatively weakly compact in $M(T)$, and hence, by the equivalence of (i) and (vi) ' of Proposition 1 and by the fact that $u^{*} x^{*}=x^{*} \circ m$, the statement (xix) holds.

Obviously, (xix) $\Rightarrow(\mathrm{xx}) \Rightarrow$ (xxi). 
(xxi) $\Rightarrow$ (i). Let $U$ be an open set in $T$ and let $\varepsilon>0$. Given an equicontinuous set $E$ in $X^{*}$, by hypothesis there exists a compact set $K \subset U$ such that $p_{E}(m(B))<\varepsilon$ for all $B \in \mathcal{B}(T)$ with $B \subset U \backslash K$. Thus $\sup _{x^{*} \in E}\left|\left(x^{*} \circ m\right)(B)\right|<\varepsilon$ for all such $B$. Since $x^{*} \circ m=u^{*} x^{*}$ and since $u^{*} E$ is bounded in $M(T)$ by Lemma 2, by the equivalence of (i) and (iii)(a)' of Proposition 1 it follows that $u^{*} E$ is relatively weakly compact in $M(T)$. Then $u$ is weakly compact by Proposition 4 .

(xix) $\Rightarrow$ (xxii). Obvious.

(xxii) $\Rightarrow$ (i). Let $K$ be compact in $T$ and $\varepsilon>0$. By hypothesis, given an equicontinuous set $E$ in $X^{*}$, there exists an open set $U$ with $K \subset U$ such that $p_{E}(m(B))<\varepsilon$ for all $B \in \mathcal{B}(T)$ with $B \subset U \backslash K$. Then by Lemma $2 u^{*} E$ is bounded, and $\sup _{x^{*} \in E}\left|\left(x^{*} \circ m\right)(B)\right|<\varepsilon$ for all such $B$. The Borel inner regularity of $m$ in $T$ implies as in the proof of (xxi) $\Rightarrow$ (i) that there exists a compact set $K$ in $T$ such that $\sup _{\mu \in u^{*} E}|\mu(B)|<\varepsilon$ for all $B \in \mathcal{B}(T)$ with $B \subset T \backslash K$. Thus by Theorem 4.22 .1 of Edwards [E], $u^{*} E$ is relatively weakly compact in $M(T)$. Now an appeal to Proposition 4 proves that $u$ is weakly compact.

Theorem 7. Let $X, u, m$ and $m_{c}$ be as in Theorem 4. Then $u$ is weakly compact if and only if any one of the following conditions holds, where the regularities referred to are with respect to the topology $\tau_{e}$ of $X^{* *}$.

(xxiii) The vector measure $m_{c}$ is $\sigma$-Borel regular.

(xxiv) The vector measure $m_{c}$ is $\sigma$-Borel inner regular.

(xxv) The vector measure $m_{c}$ is $\sigma$-Borel inner regular in each $\sigma$-Borel open set $U$ in $T$ and in the set $T$.

(xxvi) The vector measure $m_{c}$ is $\sigma$-Borel outer regular in each compact subset of $T$ and is $\sigma$-Borel inner regular in the set $T$.

Proof. Let $E$ be an equicontinuous set in $X^{*}$ and let $\varepsilon>0$.

Following an argument similar to that in the proof of (i) $\Rightarrow$ (xix) (resp. of (xxi) $\Rightarrow$ (i) with obvious modifications) and appealing to the equivalence of (i) and (vi) " (resp. of (i) and (iii) $\left\{(a)^{\prime \prime}\right.$ and $\left.(b)^{\prime \prime}\right\}$ ) of Proposition 1, and to Proposition 4, one can show that (i) $\Rightarrow$ (xxiii) (resp. (xxv) $\Rightarrow(\mathrm{i})$ ). Clearly, (xxiii) $\Rightarrow$ (xxiv).

(xxiv) $\Rightarrow(\mathrm{xxv})$. By Lemma 2 the set $u^{*} E$ is bounded in $M(T)$. The hypothesis (xxiv) then implies that $u^{*} E$ is uniformly $\sigma$-Borel inner regular, and consequently, by the equivalence of (iv) $)^{\prime \prime}$ and (iii) $\left\{(a)^{\prime \prime}\right.$ and $\left.(b)^{\prime \prime}\right\}$ of Proposition 1 the statement (xxv) holds.

(xxiii) $\Rightarrow$ (xxvi). $u^{*} E$ is bounded in $M(T)$ by Lemma 2, and the hypothesis implies that the set $u^{*} E$ is uniformly $\sigma$-Borel regular. Thus by the equivalence of (i) and (vi)" of Proposition 1 , the set $u^{*} E$ is relatively weakly compact in $M(T)$. Then by Theorem 4.22.1 of Edwards [E] and by Theorem 50.D of [Ha] the statement (xxvi) holds.

(xxvi) $\Rightarrow$ (i). By Lemma $2, u^{*} E$ is bounded in $M(T)$. Let $K$ be a compact set in $T$. Then by hypothesis there exists a $\sigma$-Borel open set $U$ in $T$ such that $K \subset U$ and

$$
\sup _{\mu \in u^{*} E}|\mu|(U \backslash K)<\varepsilon,
$$

where the variation is taken with respect to $\mathcal{B}_{c}(T)$. Then by Lemma 1 it follows that (4)(a) of Theorem 4.22.1 of [E] is satisfied by $u^{*} E$ in $K$. Again by hypothesis 
there exists a compact $C$ in $T$ such that

$$
\sup _{\mu \in u^{*} E}|\mu(B)| \leq \varepsilon
$$

for all $B \in \mathcal{B}_{c}(T)$ with $B \subset T \backslash C$. Then, for each compact $K \subset T \backslash C$ and $x^{*} \in E$, by (5.1) and Lemma 1 we have

$$
\begin{aligned}
\left|x^{*} \circ m\right|(K)=\sup \left\{\sum_{i=1}^{n}\left|\left(x^{*} \circ m\right)\left(B_{i}\right)\right|:\left(B_{i}\right)_{i=1}^{n} \subset \mathcal{B}_{c}(T),\right. & \\
B_{i} & \left.\cap B_{j}=\emptyset, i \neq j, \bigcup_{i=1}^{n} B_{i}=K\right\} \leq 4 \varepsilon .
\end{aligned}
$$

Since $\left|x^{*} \circ m\right|$ is Borel regular by Theorem 1(i), it follows that

$$
\left|x^{*} \circ m\right|(T \backslash C)=\sup _{K \text { compact }, K \subset T \backslash C}\left|x^{*} \circ m\right|(K) \leq 4 \varepsilon .
$$

Consequently, we have

$$
\sup _{\mu \in u^{*} E}|\mu|(T \backslash C) \leq 4 \varepsilon,
$$

and thus (4)(b) of Theorem 4.22.1 of [E] is also satisfied by $u^{*} E$. Therefore, $u^{*} E$ is relatively weakly compact in $M(T)$, and hence $u$ is weakly compact by Proposition 4.

Remark 5. The above theorem can be compared with Corollary 4.9 of Panchapagesan [Pa3], where the vector measure is assumed to be $\sigma$-additive on $\mathcal{B}_{c}(T)$. Since the vector measure $m$ is $\sigma$-additive only in the $\sigma\left(X^{* *}, X^{*}\right)$-topology of $X^{* *}$, unlike the said corollary, the hypothesis of $\sigma$-Borel inner regularity of $m_{c}$ in the set $T$ has to be included in the statements (xxv) and (xxvi) for the validity of their equivalence with the $\sigma$-Borel regularity.

Theorem 8. Let $X, u, m$ and $m_{o}$ be as in Theorem 4. Then $u$ is weakly compact if and only if any one of the following equivalent conditions holds, where the regularities referred to are with respect to the topology $\tau_{e}$ of $X^{* *}$.

(xxvii) The vector measure $m_{o}$ is Baire regular.

(xxviii) The vector measure $m_{o}$ is Baire inner regular.

(xxix) The vector measure $m_{o}$ is Baire inner regular in each open Baire set $U$ in $T$ and in the set $T$.

(xxx) The vector measure $m_{o}$ is Baire outer regular in each compact $G_{\delta}$ in $T$ and Baire inner regular in the set $T$.

Proof. The proof is similar to that of Theorem 7, except that we have to use Baire sets in place of $\sigma$-Borel sets in Proposition 1 and make use of Theorem 50.D of Halmos [Ha] in the last step to show that $\sup _{x^{*} \in E}\left|x^{*} \circ m\right|(T \backslash C) \leq 4 \varepsilon$. The details are left to the reader.

Remark 6. It is well known that a $\sigma$-additive lcHs-valued Baire measure is Baire regular (see [DK], [DL]). Neither this result nor the Baire measure case of Corollary 4.9 of [Pa3] can hold here verbatim, as $m$ is $\sigma$-additive only in $\sigma\left(X^{* *}, X^{*}\right)$-topology and hence the Baire regularity conditions have to be imposed on $m_{o}$ for the validity of the above theorem. 
Theorem 9. Let $X, u, m, m_{c}$ and $m_{o}$ be as in Theorem 4. Then $u$ is weakly compact if and only if anyone of the following equivalent conditions holds:

(xxxi) All bounded Borel measurable scalar functions on $T$ are $m$-integrable and $\int_{T} f d m \in X$.

(xxxii) All bounded $\mathcal{B}_{c}(T)$-measurable scalar functions on $T$ are $m_{c}$-integrable and $\int_{T} f d m_{c} \in X$.

(xxxiii) All bounded Baire measurable scalar functions on $T$ are $m_{o}$-integrable and $\int_{T} f d m_{o} \in X$.

(xxxiv) All bounded scalar functions $f$ belonging to the first Baire class in $T$ are $m_{o}$-integrable and $\int_{T} f d m_{o} \in X$.

(xxxv) $u^{* *} f \in X$ for all bounded scalar functions $f$ belonging to the first Baire class in $T$.

Proof. By Theorem 1(iv), $m$ has $\tau_{e}$-bounded range in $X^{* *}$. If $u$ is weakly compact, then by Theorem $2, m$ has range in $X$. As $X$ is quasicomplete, by the results of Section 3, (i) implies each one of the statements (xxxi)-(xxxiv). If (xxxi) (resp. (xxxii), (xxxiii)) holds, then for $A \in \mathcal{B}(T)$ (resp. $A \in \mathcal{B}_{c}(T), A \in \mathcal{B}_{o}(T)$ ), $m(A) \in X$ (resp. $\left.m_{c}(A) \in X, m_{o}(A) \in X\right)$ and consequently, by (ii) (resp. by (xiv), by (xv)) $u$ is weakly compact.

(xxxiv) $\Rightarrow$ (vii). Let $U$ be an open Baire set in $T$. Then by Proposition $2, U$ is the union of an increasing sequence of compact $G_{\delta}$ sets $K_{n}$ in $T$. Consequently, by Urysohn's lemma there exists an increasing sequence $\left(f_{n}\right)$ of nonnegative continuous functions with compact support in $T$ such that $f_{n}(t) \rightarrow \chi_{U}(t)$ for each $t \in T$. Thus $\chi_{U}$ belongs to the first Baire class in $T$, and hence by hypothesis $m_{o}(U) \in X$. Thus (vii) holds.

(xxxiv) $\Rightarrow($ xxxv). Note that the bounded scalar functions of the first Baire class belong to $C_{o}^{* *}(T)$. If (xxxiv) holds, then as (xxxiv) implies (vii) $\Leftrightarrow(\mathrm{i}), u$ is weakly compact and hence by $(\mathrm{xv}), m_{o}(A) \in X$ for each $A \in \mathcal{B}_{o}(T)$. Moreover, by Theorem 1(iv), $m_{o}$ has $\tau$-bounded range in $X$. If $f$ is a bounded Baire function, then $f$ is $m_{o}$-integrable with $\int_{T} f d m_{o} \in X$, and hence for each $x^{*} \in X^{*}$ we have

$$
\left\langle\int_{T} f d m_{o}, x^{*}\right\rangle=\int_{T} f d x^{*} \circ m_{o}=\int_{T} f d u^{*} x^{*}=\left\langle f, u^{*} x^{*}\right\rangle=\left\langle u^{* *} f, x^{*}\right\rangle .
$$

Thus $u^{* *} f \in X$. (Alternatively, one can also use Lemma 1 of $[\mathrm{G}]$.)

Consequently, (i) $\Rightarrow$ (xxxiv) $\Rightarrow($ xxxv).

$(\mathrm{xxxv}) \Rightarrow$ (vii). Since $u^{* *}\left(\chi_{U}\right)=m_{o}(U)$ for each open Baire set $U$ in $T$ and since $\chi_{U}$ belongs to the first Baire class as observed in the proof of (xxxiv) $\Rightarrow$ (vii), it follows from the hypothesis that $m_{o}(U) \in X$ for all open Baire sets $U$ in $T$. Hence (vii) holds.

Remark 7. In [Pa5] we will give further characterizations of weakly compact operators on $C_{o}(T)$ considered as weakly compact Radon operators.

\section{Applications}

As an immediate consequence of the results of Section 5 we deduce the well known results on vector measures such as the regularity (resp. regular Borel extendability) of $X$-valued $\sigma$-additive Baire measures (resp. when $X$ is quasicomplete), the strict Dunford-Pettis property of $C_{o}(T)$, and a theorem of Pelczyński [Pe] and of Thomas [Th] on weakly compact operators on $C_{o}(T)$. Also we give a generalization of Corollary VI.2.17 of [DU] to quasicomplete lcHs-valued operators on $C_{o}(T)$. 
Theorem 10. Let $X$ be a lcHs and T a locally compact Hausdorff space. Then each $X$-valued $\sigma$-additive Baire measure $\mu_{o}$ is regular. If $X$ is further quasicomplete, then there exists a unique $X$-valued $\sigma$-additive regular Borel (resp. regular $\sigma$-Borel) vector measure $\mu$ on $\mathcal{B}(T)$ (resp. $\mu_{c}$ on $\mathcal{B}_{c}(T)$ ) such that $\left.\mu\right|_{\mathcal{B}_{o}(T)}=\mu_{o}$ (resp. $\left.\left.\mu_{c}\right|_{\mathcal{B}_{o}(T)}=\mu_{o}\right)$. Besides, $\mu_{c}=\mu_{\mathcal{B}_{c}(T)}$.

Proof. Let $\hat{X}$ be the completion of $X$. Since each $f \in C_{o}(T)$ is bounded and Baire measurable, by Definition 3 the function $f$ is $\mu_{o}$-integrable and $\int_{T} f d \mu_{o} \in \hat{X}$. Let

$$
V f=\int_{T} f d \mu_{o}, f \in C_{o}(T)
$$

Then $V: C_{o}(T) \rightarrow \hat{X}$ is linear. $V$ is also continuous, since for each continuous seminorm $p$ on $\hat{X}$, by Proposition 6(ii) we have

$$
\|V f\|_{p} \leq\|f\|_{T}\left\|\mu_{o}\right\|_{p}(T), f \in C_{o}(T),
$$

and $\left\|\mu_{o}\right\|_{p}(T)<\infty$. Let $m$ be the representing measure of $V$. Then by Theorem $1, m$ is an $X^{* *}$-valued vector measure on $\mathcal{B}(T)$ such that $m$ is $\sigma$-additive in the $\sigma\left(X^{* *}, X^{*}\right)$-topology, $x^{*} m \in M(T)$ for each $x^{*} \in X^{*}$ and

$$
x^{*} V f=\int_{T} f d x^{*} m
$$

for each $f \in C_{o}(T)$. Then by (6.1), (6.2) and Proposition 6(iii) we have

$$
x^{*} V f=\int_{T} f d x^{*} \mu_{o}=\int_{T} f d\left(x^{*} m\right)_{o} \text { for } f \in C_{o}(T) \text { and for } x^{*} \in X^{*},
$$

where $\left(x^{*} m\right)_{o}=\left.\left(x^{*} m\right)\right|_{\mathcal{B}_{o}(T)}$. Then, as $\left(x^{*} m\right)_{o}$ and $x^{*} \mu_{o}$ are $\sigma$-additive scalar measures on $\mathcal{B}_{o}(T)$, by the uniqueness part of the Riesz representation theorem (Baire measure version) we conclude that $\left(x^{*} m\right)(A)=\left(x^{*} \mu_{o}\right)(A)$ for each $A \in$ $\mathcal{B}_{o}(T)$. Since $\mu_{o}$ has range in $\hat{X}$ and $m$ has range in $X^{* *}$, it follows that $m(A)=$ $\mu_{o}(A)$ for all $A \in \mathcal{B}_{o}(T)$. Consequently, by Theorem 4 the operator $V$ is weakly compact, and then by (xxvii) of Theorem 8, the Baire measure $\mu_{o}\left(=m_{o}=\left.m\right|_{\mathcal{B}_{o}(T)}\right)$ is regular (as a $\sigma$-additive Baire measure with values in $X$ ).

Moreover, by Theorem 2(ii) $m$ and $m_{c}=\left.m\right|_{\mathcal{B}_{c}(T)}$ have range in $\hat{X}$. Further, $m$ is Borel regular and $\sigma$-additive by Theorems 6 and 2, while $m_{c}$ is $\sigma$-Borel regular and $\sigma$-additive by Theorems 7 and 4 . Now we shall assume that $X$ is quasicomplete. Let $\mathcal{C}(E)=\{K: K \subset E, K$ compact $\}$ for $E \in \mathcal{B}(T)$. Let us define $K_{1} \leq K_{2}$ for $K_{1}, K_{2} \in \mathcal{C}(E)$ if $K_{1} \subset K_{2}$. Similarly, let $\mathcal{U}(E)=\{O: E \subset O, O$ open $\}$ and let us define $O_{1} \leq O_{2}$ for $O_{1}, O_{2} \in \mathcal{U}(E)$ if $O_{2} \subset O_{1}$. Let $\mathcal{U}_{o}(E)=\mathcal{B}_{o}(T) \bigcap \mathcal{U}(E)$ with the same partial ordering as in $\mathcal{U}(E)$. Then clearly $\mathcal{C}(E), \mathcal{U}(E)$ and $\mathcal{U}_{o}(E)$ are directed sets. Given a balanced closed neighborhood $W$ of 0 in $\hat{X}$ and a compact set $K$ in $T$, by the Borel regularity of $m$ there exists $O_{o} \in \mathcal{U}(K)$ such that $m(K)-m(O) \in W$ for all $O \in \mathcal{U}(K)$ with $O \geq O_{o}$. Then in virtue of Theorem 50.D of Halmos [Ha], we have $m(K)-m(U) \in W$ for all open Baire sets $U$ in $T$ with $K \subset U \subset O_{o}$. Thus we have

$$
m(K)=\lim _{U \in \mathcal{U}_{o}(K)} m(U)=\lim _{U \in \mathcal{U}_{o}(K)} m_{o}(U) \in X,
$$

since the range of $m_{o}$ is bounded in $X$ and $X$ is quasicomplete. Consequently, the inner Borel regularity of $m$ in $E \in \mathcal{B}(T),(6.3)$, the $\tau$-boundedness of $\{m(K): K \in$ $\mathcal{C}(T)\}$ and the quasicompleteness of $X$ imply that $m(E) \in X$ for each $E \in \mathcal{B}(T)$. Therefore, the ranges of $m$ and $m_{c}$ are contained in $X$. This shows that $\mu_{o}$ has a 
$\sigma$-additive regular Borel (resp. $\sigma$-Borel) extension $m$ (resp. $m_{c}$ ) with values in $X$, and moreover, $m_{c}=\left.m\right|_{\mathcal{B}_{c}(T)}$.

Finally, to prove the uniqueness of $m$ (resp. of $m_{c}$ ), let $m_{1}$ (resp. $m_{2}$ ) be an $X$-valued Borel regular (resp. $\sigma$-Borel regular) $\sigma$-additive extension of $\mu_{o}$. Then, as $V f=\int_{T} f d \mu_{o}, f \in C_{o}(T)$, it follows that

$$
x^{*} V f=\int_{T} f d x^{*} \mu_{o}=\int_{T} f d x^{*} m_{1}=\int_{T} f d x^{*} m_{2}=\int_{T} f d x^{*} m=\int_{T} d x^{*} m_{c}
$$

for $f \in C_{o}(T)$ and $x^{*} \in X^{*}$. Now by the uniqueness part of the Riesz representation theorem in the Borel version (resp. $\sigma$-Borel version), we conclude that $x^{*} m_{1}=x^{*} m$ (resp. $x^{*} m_{2}=x^{*} m_{c}$ ) for each $x^{*} \in X^{*}$. Then by the Hahn-Banach theorem, $m_{1}=m$ (resp. $m_{2}=m_{c}$ ). Define $\mu=m$ and $\mu_{c}=m_{c}$.

Remark 8 . The regularity of a lcHs-valued $\sigma$-additive Baire measure on a locally compact Hausdorff space was first proved by Dinculeanu and Kluvánek in [DK], and a direct proof was later given by Dinculeanu and Lewis in [DL]. Using the results of $[\mathrm{DK}]$, Kluvánek [K] proved that each Banach space valued $\sigma$-additive Baire measure on such spaces has a unique $\sigma$-additive Borel regular extension. Kluvánek's result can easily be extended to quasicomplete lcHs-valued Baire measures. The idea of the present proof has its origin in the proof of Theorem 3.7 of Panchapagesan [Pa1]. Moreover, the last part of the above theorem generalizes Theorem 3.7 of [Pa1] and Theorem 2.4 of $[\mathrm{Pa} 2]$ to vector valued $\sigma$-additive Baire measures.

Theorem 11. $C_{o}(T)$ has the strict Dunford-Pettis property (briefly, (SDP)property). That is, for each weakly compact operator $u: C_{o}(T) \rightarrow X, X$ a quasicomplete lcHs, u transforms each weak Cauchy sequence in $C_{o}(T)$ into a convergent sequence in $X$, and consequently, $u$ transforms each weakly compact set in $C_{o}(T)$ into a relatively compact set in $X$ if $X$ is moreover metrizable.

Proof. If $\left(f_{n}\right)_{n=1}^{\infty}$ is weakly Cauchy in $C_{o}(T)$, then $\left(f_{n}\right)$ converges to a function $f$ pointwise in $T$ and $\left(f_{n}\right)_{n=1}^{\infty}$ is norm bounded. By Theorem 2 the representing measure $m$ of $u$ has range in $X$ and is $\sigma$-additive in $X$. Consequently, by Definition 3, Theorem 1(iii), Proposition 7, and the Hahn-Banach theorem we have

$$
\lim _{n} u f_{n}=\lim _{n} \int_{T} f_{n} d m=\int_{T} f d m \in X .
$$

Hence $C_{o}(T)$ has the (SDP)-property. The second part is immediate from the first due to the Smulian theorem (Theorem 8.12.1 of Edwards [E]).

Remark 9. The above result is due to Grothendieck [G], and was used in the proof of Theorem 6 of $[\mathrm{G}]$ (which treats only the compact case). The proofs of all the characterization theorems given in Section 5 are independent of the (SDP)-property of $C_{o}(T)$, and our proof of the above theorem is quite analogous to that of Corollary 3.3 of $[\mathrm{BDS}]$.

The following theorem is a generalization of Corollary VI.2.17 of [DU] to quasicomplete lcHs-valued operators on $C_{o}(T)$.

Theorem 12. Let $u: C_{o}(T) \rightarrow X$ be a continuous linear map and let $X$ be a quasicomplete lcHs. Then the following are equivalent:

(i) $u$ is unconditionally convergent; that is, u maps weakly unconditionally covergent Cauchy series into unconditionally convergent series. 
(ii) $u$ is weakly compact.

(iii) $u$ maps sequences that tend to zero weakly into sequences convergent to zero.

(iv) u maps weak Cauchy sequences into convergent sequences.

(v) If $\left(f_{n}\right)$ is a bounded sequence in $C_{o}(T)$ with $f_{n} . f_{\ell}=0$ for $n \neq \ell$, then $\lim _{n} u\left(f_{n}\right)=0$.

Proof. (i) $\Rightarrow$ (ii). Let $U$ be an open Baire set. Then by Proposition 2, $U$ is of the form $U=\bigcup_{n=1}^{\infty} K_{n}$, with $K_{n}$ compact $G_{\delta}$ for each $n$ and $K_{n} \nearrow$. By Urysohn's lemma there exists an increasing sequence $\left(g_{n}\right)$ of nonnegative continuous functions with compact support such that $g_{n}(t) \nearrow \chi_{U}(t)$ for each $t \in T$. Then by the Lebesgue dominated convergence theorem,

$$
\mu(U)=\lim _{n} \int_{T} g_{n} d \mu=\lim _{n} \sum_{k=1}^{n}\left(\int_{T} g_{k+1} d \mu-\int_{T} g_{k} d \mu\right)+\int_{T} g_{1} d \mu
$$

for each $\mu \in M(T)$. Moreover,

$$
\begin{aligned}
& \sum_{n=1}^{\infty}\left|\int_{T}\left(g_{n+1}-g_{n}\right) d \mu\right| \leq \sum_{n=1}^{\infty} \int_{T}\left(g_{n+1}-g_{n}\right) d|\mu| \\
& \quad=\lim _{k} \int_{T} g_{k} d|\mu|-\int_{T} g_{1} d|\mu| \leq 2|\mu|(U)<\infty .
\end{aligned}
$$

Therefore the series $\sum_{n=1}^{\infty}\left(g_{n+1}-g_{n}\right)+g_{1}$ is an unconditionally weakly Cauchy series (see p.150 of [DU]), and hence by (i), there exists a vector $x \in X$ such that

$$
\sum_{n=1}^{\infty}\left(u\left(g_{n+1}\right)-u\left(g_{n}\right)\right)+u\left(g_{1}\right)=\lim _{n} u\left(g_{n}\right)=x .
$$

If $m$ is the representing measure of $u$, then by Theorem 1(iii) and by the Lebesgue dominated convergence theorem we have

$$
x^{*} m(U)=\lim _{n} x^{*} u\left(g_{n}\right)=x^{*} x
$$

for each $x^{*} \in X^{*}$, and hence $m(U)=x \in X$. Consequently, $u$ is weakly compact by Theorem 3(vii).

(ii) $\Rightarrow$ (i) and (iv) by Theorem 11, and (iv) $\Rightarrow$ (iii) obviously. (One can also apply the Orlicz-Pettis theorem to show (ii) $\Rightarrow$ (i) as on p.150 of [DU].)

(iii) $\Rightarrow(\mathrm{v})$. Such a norm bounded sequence $\left(f_{n}\right)$ converges to zero pointwise in $T$ and hence by the Lebesgue bounded convergence theorem is weakly convergent to zero. Thus (iii) implies (v).

(v) $\Rightarrow$ (ii). If $u$ is not weakly compact and if $m$ is the representing measure of $u$, then by Propositions 1(ii) and 4 there would exist an equicontinuous set $E$ in $X^{*}$, an $\varepsilon>0$, a disjoint sequence $\left(U_{n}\right)$ of open Baire sets in $T$ and a sequence $\left(x_{n}^{*}\right)$ in $E$ such that $\left|\left(x_{n}^{*} \circ m\right)\left(U_{n}\right)\right|>\varepsilon$. By Propositions 2 and 7 and by Urysohn's lemma we can choose a continuous function $f_{n}$ with compact support such that $0 \leq f_{n} \leq \chi_{U_{n}}$ and

$$
\left|\int_{T} f_{n} d\left(x_{n}^{*} \circ m\right)\right|>\varepsilon
$$

for each $n$. Then clearly $\left(f_{n}\right)$ satisfies the hypothesis of $(\mathrm{v})$, and consequently $\lim _{n} u\left(f_{n}\right)=0$. Thus there exists $n_{o}$ such that $p_{E}\left(u\left(f_{n}\right)\right)=\sup _{x^{*} \in E}\left|x^{*}\left(u\left(f_{n}\right)\right)\right|<\varepsilon$ for all $n \geq n_{o}$. Then by Theorem 1 (iii), this means that $\sup _{x^{*} \in E}\left|\int_{T} f_{n} d x^{*} m\right|<\varepsilon$ for $n \geq n_{o}$, which contradicts (6.4). Hence $u$ is weakly compact. 
Theorem 13. Suppose the quasicomplete lcHs $X$ contains no copy of $c_{o}$. Then every continuous linear map $u: C_{o}(T) \rightarrow X$ is weakly compact. If $\omega$ is the set $\mathbb{N}$ endowed with the discrete topology and if $X$ is a quasicomplete lcHs such that every continuous linear map $u: C_{o}(\omega) \rightarrow X$ is weakly compact, then $X$ contains no copy of $c_{o}$. Consequently, a quasicomplete lcHs $X$ contains no copy of $c_{o}$ if and only if each continuous linear map $u: C_{o}(T) \rightarrow X$ is weakly compact for every locally compact Hausdorff space T.

Proof. Suppose $X$ contains no copy of $c_{o}$ and suppose $u: C_{o}(T) \rightarrow X$ is a continuous linear map. Let $m$ be the representing measure of $u$. Given an open Baire set $U$ in $T$, let $\left(K_{n}\right)$ and $\left(g_{n}\right)$ be chosen as in the proof of (i) $\Rightarrow$ (ii) of Theorem 12 . Then by the Lebesgue dominated convergence theorem we have

$$
x^{*} m(U)=\lim _{n} \int_{T} g_{n} d x^{*} m=\sum_{n=1}^{\infty} \int_{T}\left(g_{n+1}-g_{n}\right) d x^{*} m+\int_{T} g_{1} d x^{*} m
$$

for each $x^{*} \in X^{*}$. Since $g_{n+1}-g_{n} \geq 0$ for each $n$, it follows that

$$
\begin{aligned}
& \sum_{n=1}^{\infty}\left|\int_{T}\left(g_{n+1}-g_{n}\right) d x^{*} m\right| \leq \sum_{n=1}^{\infty} \int_{T}\left(g_{n+1}-g_{n}\right) d\left|x^{*} m\right| \\
& \quad=\lim _{n} \int_{T} g_{n+1} d\left|x^{*} m\right|-\int_{T} g_{1} d\left|x^{*} m\right| \leq 2\left|x^{*} m\right|(U)<\infty .
\end{aligned}
$$

On the other hand, by Theorem 1(iii), $\int_{T}\left(g_{n+1}-g_{n}\right) d x^{*} m=x^{*} u\left(g_{n+1}-g_{n}\right)$, and hence it follows that $\sum_{n=1}^{\infty}\left|x^{*}\left(u\left(g_{n+1}\right)-u\left(g_{n}\right)\right)\right|<\infty$ for each $x^{*} \in X^{*}$. Since $X$ contains no copy of $c_{o}$, by Theorem 4 of Tumarkin [Tu] we conclude that $\sum_{n=1}^{\infty}\left(u\left(g_{n+1}\right)-u\left(g_{n}\right)\right)$ is convergent in the topology of $X$ and thus $\lim _{n} u\left(g_{n}\right)=x$ exists in $X$. Then $x^{*} m(U)=\lim _{n} \int_{T} g_{n} d x^{*} m=\lim _{n} x^{*} u\left(g_{n}\right)=x^{*} x$ for each $x^{*} \in X^{*}$, and consequently $m(U)=x \in X$. Therefore, $u$ is weakly compact by Theorem 3(vii).

Suppose each continuous linear map $u: C_{o}(\omega) \rightarrow X$ is weakly compact, and suppose $\tau$ is the topology of $X$. Let $\left(x_{n}\right)$ be a sequence of vectors in $X$ such that $\sum_{n=1}^{\infty}\left|x^{*}\left(x_{n}\right)\right|<\infty$ for each $x^{*} \in X^{*}$. Let us define $u\left(\chi_{\{n\}}\right)=x_{n}$ for each $n$ and extend $u$ linearly on the set $S$ of all scalar functions of the form $\sum_{i=1}^{k} \alpha_{i} \chi_{\left\{n_{i}\right\}}$, where $S$ is endowed with the supremum norm. By hypothesis on $\left(x_{n}\right)$, the set $\left\{u f: f \in S,\|f\|_{\mathbb{N}} \leq 1\right\}$ is weakly bounded in $X$ and hence is $\tau$-bounded. Then by Theorem 1.32 of Rudin [R], $u$ is continuous on $S$. Since $S=C_{c}(\omega)$ is norm dense in $C_{o}(\omega)$ and $X$ is quasicomplete, $u$ admits a unique continuous linear extension to the whole of $C_{o}(\omega)$; let us denote the extension too by $u$. Then by hypothesis $u$ is weakly compact. Let $m$ be the representing measure of $u$. Since $\left.u^{* *}\right|_{C_{o}(\omega)}=u$, it follows that $m(F)=u\left(\chi_{F}\right)=\sum_{n \in F} x_{n}$, for each finite set $F \subset \mathbb{N}$. Since $u$ is weakly compact, by Theorem 2(ii) the range of $m$ is contained in $X$; and by Theorem $6(\mathrm{xx}), m$ is Borel inner regular. Thus, given a subset $U$ of $\mathbb{N}, \varepsilon>0$ and a $\tau$-continuous seminorm $p$ on $X$, there exists a finite set $F \subset U$ such that

$$
p\left(m(U)-\sum_{n \in G} x_{n}\right)<\varepsilon
$$

for all finite sets $G$ with $F \subset G \subset U$. Therefore, $m(U)=\sum_{n \in U} x_{n}$, where the series is unconditionally $\tau$-convergent in $X$. In particular, $\sum_{n=1}^{\infty} x_{n}$ is unconditionally $\tau$ convergent; and thus by Theorem 4 of Tumarkin [Tu], $X$ contains no copy of $c_{o}$. 
Remark 10. The first part of the above theorem was proved by Pelczyński [Pe] when $T$ is compact and $X$ is a Banach space, and was later extended by Thomas [Th] for the general case in an equivalent form. Both proofs use the technique of reduction to the compact metrizable case. Thanks to our Theorem 3(vii) we are able to provide a direct proof without such a reduction. The second part of the above theorem is essentially Theorem 5.3 (bounded Radon measure case) of Thomas [Th], where the proof is based on the theory of Radon vector measures.

Added in proof. In a recent paper submitted for publication, the author has given an alternative proof to obtain the said characterizations of weakly compact operators on $C_{0}(T)$. The new proof is based on the regular Borel extension of $\sigma$-additive $X$-valued Baire measures on $T$.

\section{REFERENCES}

[BDS] R.G. Bartle, N. Dunford, and J.T. Schwartz, Weak compactness and vector measures, Canad. J.Math. 7, (1955), 289-305. MR 16:1123c

[DU] J. Diestel, and J.J. Uhl, Vector Measures, Survey No.15, Amer. Math. Soc. Providence, R.I., (1977). MR 56:12216

[Di] N. Dinculeanu, Vector Measures, Pergamon Press, New York, (1967). MR 34:6011

[DK] N. Dinculeanu, and I. Kluvánek, On vector measures, Proc. London Math. Soc. 17, (1967), 505-512. MR 35:5571

[DL] N. Dinculeanu, and P.W. Lewis, Regularity of Baire measures, Proc. Amer. Math. Soc. 26, (1970), 92-94. MR 41:5588

[Do] I. Dobrakov, On integration in Banach spaces, IV, Czech. Math. J. 30, (1980), 259-279. MR 81m:28006

[E] R. E. Edwards, Functional Analysis, Theory and Applications, Holt, Rinehart and Winston, New York, (1965). MR 36:4308

[G] A. Grothendieck, Sur les applications linéares faiblement compactes d'espaces du type $C(K)$, Canad. J. Math. 5, (1953), 129-173. MR 15:438b

[Ha] P. R. Halmos, Measure Theory, Van Nostrand, New York, (1950). MR 11:504d

[Ho] J. Horváth, Topological Vector Spaces and Distributions, Addison-Wesley, Reading, (1966). MR 34: 4863

[K] I. Kluvánek, Characterization of Fourier-Stieltjes transforms of vector and operator valued measures, Czech. Math. J. 17, (1967), 261-277. MR 37:6430

[M] C. W. McArthur, On a theorem of Orlicz and Pettis, Pacific J. Math., 22, (1967), 297-302. MR 33:4702

[Pa1] T.V. Panchapagesan, On complex Radon measures I, Czech. Math. J. 42, (1992), 599-612. MR 94e: 28014

[Pa2] — On complex Radon measures II, Czech. Math. J. 43, (1993), 65-82. MR 94i:28011

[Pa3] - Abstract regularity of additive and $\sigma$-additive group-valued set functions, Math. Slovaca, 45, (1995), 381-393. MR 97c:28027

$[\mathrm{Pa} 4]$, Baire and $\sigma$-Borel characterizations of weakly compact sets in $C_{o}(T)$, Trans. Amer. Math. Soc. 350 (1998), 4839-4847.

[Pa5] _ On Radon vector measures I, submitted.

[Pe] A. Pelczyński, Projections in certain Banach spaces, Studia Math. 19, (1960), 209-228. MR 23:A3441

[R] W. Rudin, Functional Analysis, McGraw-Hill, New York, (1973). MR 51:1315

[Th] E. Thomas, L'intégration par rapport a une mesure de Radon vectorielle, Ann. Inst. Fourier (Grenoble) 20, (1970), 55-191. MR 57:3348

[Tu] Ju. B. Tumarkin, On locally convex spaces with basis, Soviet Math. Dokl. 11, (1970), 1672-1675. MR 42:6575

Departamento de Matemáticas, Facultad de Ciencias, Universidad de los Andes, MÉRida, Venezuela

E-mail address: panchapa@ciens.ula.ve 